\title{
7 \\ The Skeletomotor System and Functional Somatic Symptoms
}

\begin{abstract}
In this chapter we continue our exploration of the neurobiology of functional somatic symptoms by considering the skeletomotor system. When the stress system activates, the skeletomotor system activates in tandem, to prepare the body for protective action. And when protective action is no longer necessary, the skeletomotor system typically deactivates. But if the child has faced severe stress of one kind or another, with the consequence that stress-system activation is maintained, activation of the skeletomotor system may also be maintained even though there is no immediate need. This maladaptive activation of the skeletomotor system may express itself in aberrant motor patterns. Some of these patterns are easily recognizable as body responses to threat that have somehow remained activated even in the absence of current threat. Other motor patterns mimic neurological conditions and result in significant functional impairment. In this chapter we also
\end{abstract}

Electronic supplementary material The online version of this chapter (https://doi.org/10.1007/978-3-030-46184-3_7) contains supplementary material, which is available to authorized users. 
introduce the reader to the fascia system. The fascia envelops and supports skeletal muscle (and other organs and tissues) and plays an important role in symptoms involving the skeletomotor system.

The skeletomotor system includes skeletal muscles, the motor nerves that innervate these muscles, and the motor-processing regions in the brain that control all aspects of motor function. Skeletal muscles are made up of striated muscle fibres (cells) that attach to the bones of the body. These muscles enable the body to move and to address, through action, the challenges of daily life. Anatomists refer to the motor nerves that innervate these muscles as somatomotor nerves. The word soma comes from the Greek and means body (or body proper). The skeletomotor system manages motor activation of the body (soma) as a whole. By contrast, as we saw in Chapter 6, the efferent nerves of the autonomic nervous system, known as visceromotor nerves, innervate heart muscle and smooth muscle-which is found in body organs and tissues (e.g., blood vessels and glands) — and also control increases in body arousal. For the origins and anatomy of different types of muscles, see Online Supplement 7.1.

Because functional symptoms pertaining to the skeletomotor system frequently involve pain, we must also mention the afferent nerveswhich are part of the interoceptive system-that carry information about body state (including pain) from skeletal muscle to the spinal cord and then to the brain. These afferent interoceptive nerves play a key role in chronic musculoskeletal pain. Locally, in chronic pain the endings of these nerves may release pro-inflammatory molecules in skeletal muscle (called neurogenic inflammation). The nerves themselves (separate from the activity at their endings) carry interoceptive and pain information from muscle to the spinal cord. In the spinal cordwhich is part of the central nervous system-these same nerves release pro-inflammatory molecules, resulting in central sensitization, a state in which pain signals are maintained and amplified. In turn, this central sensitization (in the spinal cord) activates nerves carrying pain signals through the spinal cord to the brain, where they help maintain activation of pain maps and the subjective experience of pain. For further discussion of pain and pain maps, see Chapters 9 and 11. 
Functional somatic symptoms associated with the skeletomotor system are wide ranging. Individual children (including adolescents) have their own characteristic patterns of skeletomotor response to stress. One simple way of conceptualizing this idea, which is used to organize the present chapter, is to borrow a notion introduced by Leah Helou and colleagues (2018), who refer to 'laryngeal muscle responders': individuals who respond to stress by tightening up the laryngeal muscles that make up the voice box. By the same token, and as discussed in this chapter, some children respond to stress by activating their postural muscles; others activate their respiratory muscles; others the muscle groups involved in actions such as coughing; and so on. Importantly for our purposes, each of these different patterns, when excessive, maladaptive, or aberrant, can present as functional somatic symptoms. But just how any particular pattern of symptoms is classified or described will depend upon the medical professional initially consulted. For example, excessive activation of laryngeal muscles may be called functional/habit/psychogenic cough (if presenting with a cough to a respiratory physician), muscle tension dysphonia (if presenting with loss of voice to a speech pathologist), functional dysphagia (if presenting with concurrent difficulties in swallowing to a gastroenterologist), muscle tension dysphagia (if presenting with difficulties in swallowing to an otorhinolaryngologist), or functional tic disorder/functional neurological disorder (FND) (if presenting with vocal noises to a neurologist). For references see Online Supplement 1.3.

\section{The Skeletomotor System Works Together with the Autonomic Nervous (Visceromotor) System}

Activation of the skeletomotor system and activation of the autonomic nervous system occur hand in hand. The two systems function as coupled systems (Koizumi and Brooks 1972; Dum et al. 2016; Jafari et al. 2017) (see Fig. 7.1). They work together as an integrated whole. This collaboration begins on the brain system level and flows through to the body system level. Activation of the autonomic system-in particular, an increase 


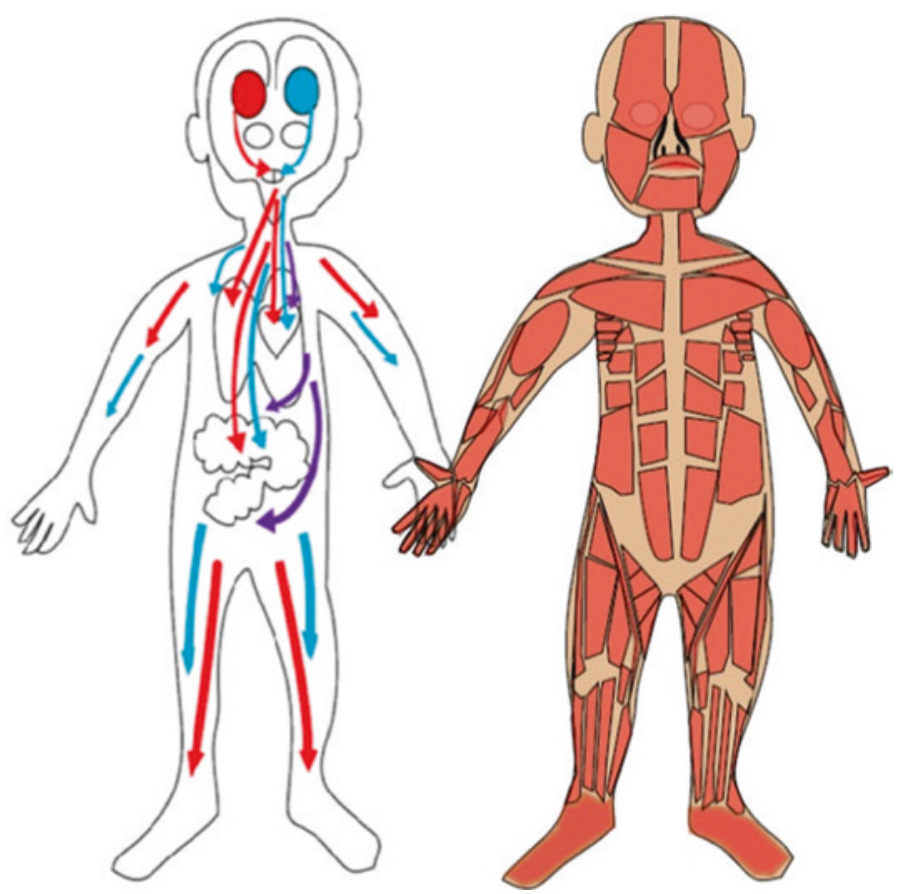

Fig. 7.1 The autonomic and skeletomotor systems as coupled systems. The figure on the left represents the efferent (motor) component of the autonomic system. The sympathetic nerves are depicted in red. The restorative parasympathetic nerves are depicted in blue. The defensive parasympathetic nerves are depicted in purple. The figure on the right represents the skeletomotor system. The holding of hands represents the coupling between the two systems. The autonomic system-also known as the visceromotor system-activates to mediate increases in body arousal and motor changes in the organs and tissues. The skeletomotor system activates to mediate an increase in breathing rate (to keep up with the body's increased need for oxygen for producing energy), to increase tone in skeletal muscles to prepare the body for action, and to generate movement. The systems work together as an integrated whole (๑ Kasia Kozlowska 2017)

in sympathetic arousal-is typically accompanied by activation of the skeletomotor system, and vice versa. Likewise, when one of these systems becomes excessively activated or dysregulated, the other does so, too. 
Because of this coupling between systems, some therapists refer to coupled activation of the autonomic nervous system and skeletal muscle as tension patterns (Baker 2017) or as stress-related bracing or patterns of constriction or contraction (see Online Supplement 7.1). Body-oriented psychotherapists_-such as those working from Peter Levine's Somatic Experiencing, Patricia Ogden's Sensorimotor Psychotherapy, or Kathy Kain's use of therapeutic touch — use tracking and settling/release of autonomic/skeletal muscle/fascia activation patterns as their primary therapeutic tools (Levine 1997; Payne et al. 2015; Ogden and Fisher 2015; Kain and Terrell 2018).

\section{Brain Stress Systems and Skeletomotor Function}

While this chapter focuses on the body expression of aberrant motor symptom patterns, it is important to highlight that all of the symptom patterns described below also involve, and are the result of, excessive activation of the brain stress systems (for further discussion see Chapter 11). That is, excessive activation of the brain stress systems appears either (1) to maintain activation of motor-processing regions that are associated with and generate specific aberrant motor patterns or (2) to disrupt motor processing in the brain, generating unusual motor patterns that mimic neurological diseases (and are diagnosed as FND).

\section{Symptoms Associated with Muscles That Stabilize Body Posture}

Sympathetic activation and its state of high arousal are typically coupled with increased tone in postural muscles, which stabilize body posture and ensure that the body is prepared for action. Increases in tone involve activation of both skeletal motor units (detectable on electromyogram) and muscle spindles (detectable with needle microelectrodes) (for muscle spindles and pain, see Online Supplement 7.1). Increased tone in postural muscles also occurs in stressful situations for which actions either are not required or cannot actually be taken effectively. Such situations 
include those whose origins are psychological (e.g., anticipatory anxiety, catastrophizing, traumatic memories), interpersonal (e.g., conflicts within relationships), or even intellectual (e.g., difficult cognitive tasks).

For example, a child may have an urge to punch a bully in the face, but this type of physical, protective action is generally deemed inappropriate in the current cultural context, and is typically inhibited. The upshot is that the body will still activate the motor action pattern (the skeletal muscles preparing for action, including the punch) but without the follow-up action — the punch itself — taking place.

Mild increases in muscle tone involving particular action patterns in response to psychological, interpersonal, or intellectual stress are common and will generally abate relatively quickly. But persistent or recurring increases in tone can be maladaptive (Westgaard et al. 2013) and may result in pain or sensations of tension in the head, neck, back, hamstring, and calf muscles. In children, this sort of excessive musculoskeletal tension can usually be identified as tenderness, trigger points, and increased muscle tension on palpation (Simons et al. 1999). Such increases in tone-the patterned activation of muscles - will typically contribute to symptoms of fatigue. And if the activation of these muscles is recurrent and ongoing, all the factors that are associated with, and that maintain, chronic pain potentially come into play (see above and Chapters 9 and 11).

Greta, a 17-year-old girl in the final year of high school, was hoping to become a doctor; her study schedule was often gruelling. After any protracted period of studying, Greta suffered from tension and pain in the muscles that support posture: the muscles of her neck, shoulders, and legs (hamstrings and calves). At night the hamstring muscle on the right throbbed with pain.

\section{Symptoms Arising in Connection with Muscles of the Respiratory Motor System: Hyperventilation}

'Hyperventilation is a respiratory stress response that occurs most typically in situations of uncontrollable stress, fear, and pain' (Jafari et al. 2017, p. 997). In states of arousal, sympathetic activation is coupled 
with increases in respiratory rate to ensure an increase in the oxygen $\left(\mathrm{O}_{2}\right)$ supply to cells for the production of energy-a process known as cellular respiration (see section on cellular respiration in Online Supplement 7.1). Carbon dioxide $\left(\mathrm{CO}_{2}\right)$, the waste product of cellular respiration, diffuses into the blood and is then eliminated by the lungs. The homeostatic range for arterial $\mathrm{CO}_{2}$ is $36-52 \mathrm{~mm} \mathrm{Hg}$ (up to $52 \mathrm{~mm}$ $\mathrm{Hg}$ in sleep). If the arterial $\mathrm{CO}_{2}$ is below the homeostatic range-the gold-standard test for hyperventilation-then the child is, by definition, hyperventilating. That is, the child is breathing faster than needed by metabolic demand, faster than required to eliminate the $\mathrm{CO}_{2}$ produced by cellular respiration. In such circumstances-with activation of the respiratory motor system (skeletal muscles that mediate respiration) exceeding the body's actual oxygen need-functional somatic symptoms may arise.

Transcutaneous monitors - a small probe placed on the finger-now enable researchers to measure arterial $\mathrm{CO}_{2}$ noninvasively by its diffusion across the skin. Arterial $\mathrm{CO}_{2}$ is denoted as $\mathrm{pCO}_{2}$ : the $\mathrm{p}$ refers to the partial pressure of carbon dioxide in the arterial blood when measured by a transcutaneous monitor.

In clinical practice, where transcutaneous monitors are not usually available, respiratory rate-with the guidance from reference ranges (Fleming et al. 2011, p. 3636) — is used as an approximate measure of hyperventilation. Very high resting-state respiratory rates ( $>25$ breaths per minute in children 9 years and older; see following vignette) fall above the 99th percentile and are a clear indictor of an overactivated respiratory motor system-and hyperventilation. High breathing rates ( $\geq 21$ breaths per minute for children $8-11$ years and $\geq 19$ breaths per minute for adolescents $12-15$ years) fall above the 75 th percentile and are highly suggestive of an overactivated respiratory motor system and hyperventilation (Kozlowska et al. 2017; Chudleigh et al. 2019).

Twelve-year-old Bella presented to the emergency department with a recurrence of non-epileptic seizures (NES) and an increase in the frequency of her fainting episodes in the context of long-term diagnoses of postural orthostatic tachycardia syndrome (POTS) and irritable bowel syndrome. The emergency doctor observed hyperventilation before 
and during the NES, with respiratory rates of over 40 breaths per minute. The video electroencephalogram (EEG) tracing was normal. The transcutaneous probe that measured arterial $\mathrm{CO}_{2}$ showed that Bella was already hyperventilating in the resting state $\left(\mathrm{pCO}_{2}\right.$ of $34 \mathrm{~mm} \mathrm{Hg}$, before Bella was instructed to hyperventilate as part of the hyperventilation challenge). During the hyperventilation component itself-when the technician used a pinwheel to help Bella hyperventilate-Bella's head dropped, and she became unresponsive, dropping the pinwheel at $25 \mathrm{~mm}$ $\mathrm{Hg}$ (a pattern that replicated some of her NES). During the 15-minute recovery period, Bella failed to return to homeostasis (see Fig. 7.2). Her resting-state heart rate was frequently $\geq 90$ beats per minute (above the 75th percentile), and her resting heart rate variability (a measure of calmness) on the biofeedback device used by the team was low (reflecting low restorative parasympathetic activity). Taken together, the biomarkers suggested overactivation of the respiratory motor system coupled with overactivation of the autonomic nervous system.

Hyperventilation and low $\mathrm{pCO}_{2}$ are associated with a cascade of neurophysiological changes (for the neurophysiology of hyperventilation, see Online Supplement 7.1). As with other biological responses, the pattern of each child's individual response to hyperventilation varies from one child to another (see Fig. 7.3 picturing children with different responses). What this means clinically is that some children experience, for example, florid symptoms in response to hyperventilation, whereas some children do not. It also means that many patterns of presentation can be encountered clinically, with different children presenting with different symptom patterns involving different body systems. Clinicians therefore need to keep in mind the entire spectrum of hyperventilation-related symptoms and to be able to identify these symptoms in any particular child, whether the symptoms occur in isolation (e.g., hyperventilation-induced chest pain) or alongside other hyperventilation-induced symptoms (e.g., hyperventilation-induced chest pain plus changes in consciousness). In addition, children can present with chronic hyperventilation combined with sudden, time-limited periods of hyperventilation (like Bella), or they can present with such time-limited increases alone, in which case their respiratory rates (and arterial $\mathrm{CO}_{2}$ ) in the relaxed, resting state may be normal. 


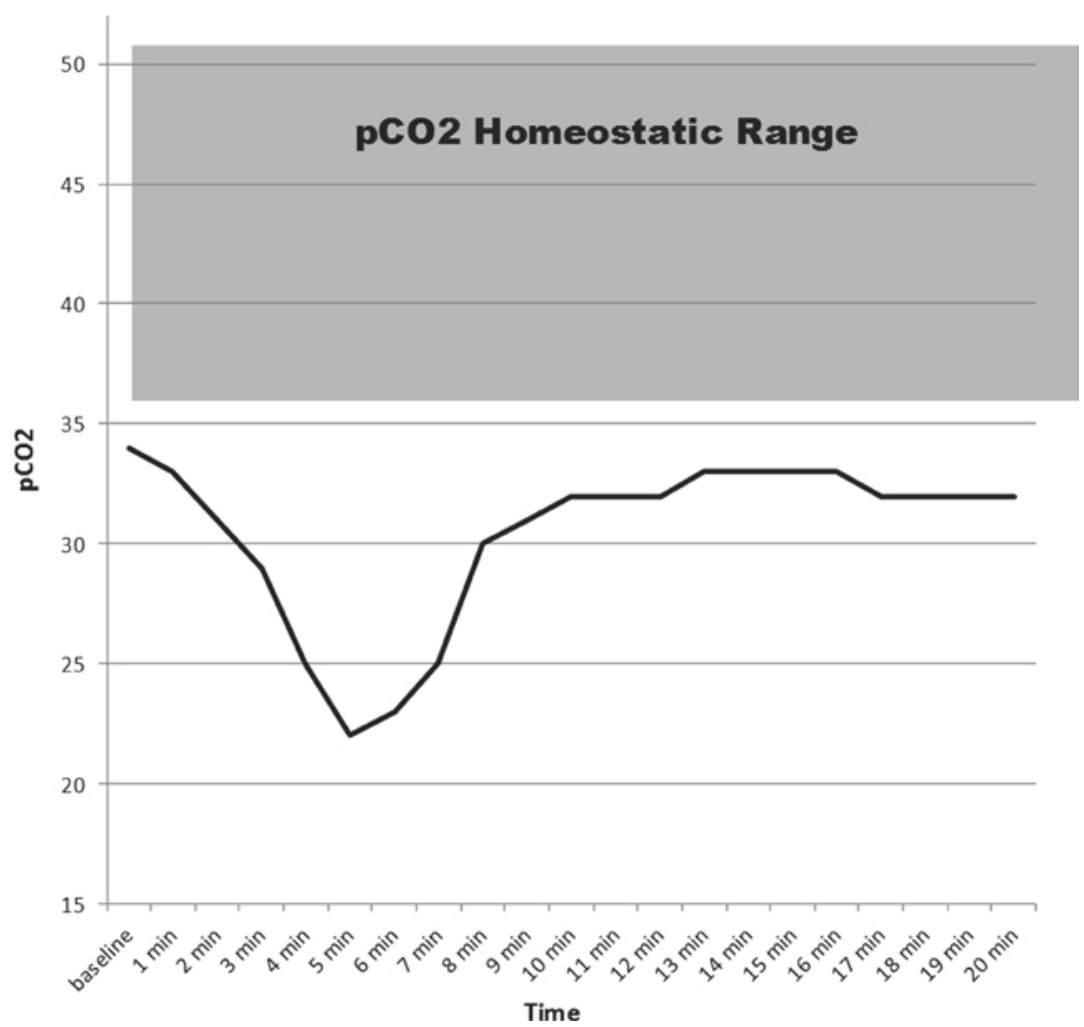

Fig. 7.2 Bella's hyperventilation challenge. This figure shows that Bella was hyperventilating at baseline, even before the EEG technician asked her to hyperventilate. She then dropped her $\mathrm{pCO}_{2}$ further (as expected) but was unable to reach the homeostatic range-or even her baseline level-after a 15-minute recovery period (@ Kasia Kozlowska 2017)

Hyperventilation is associated with major changes in both the body generally (not just the respiratory system) and the brain.

\section{In the Body}

Hyperventilation causes sensory and motor nerves to become activated. Excessive activation of nerves may cause pins and needles in the hands and feet, twitchy muscles, or even spasms in the hands or feet 


\section{When I hyperventilate}
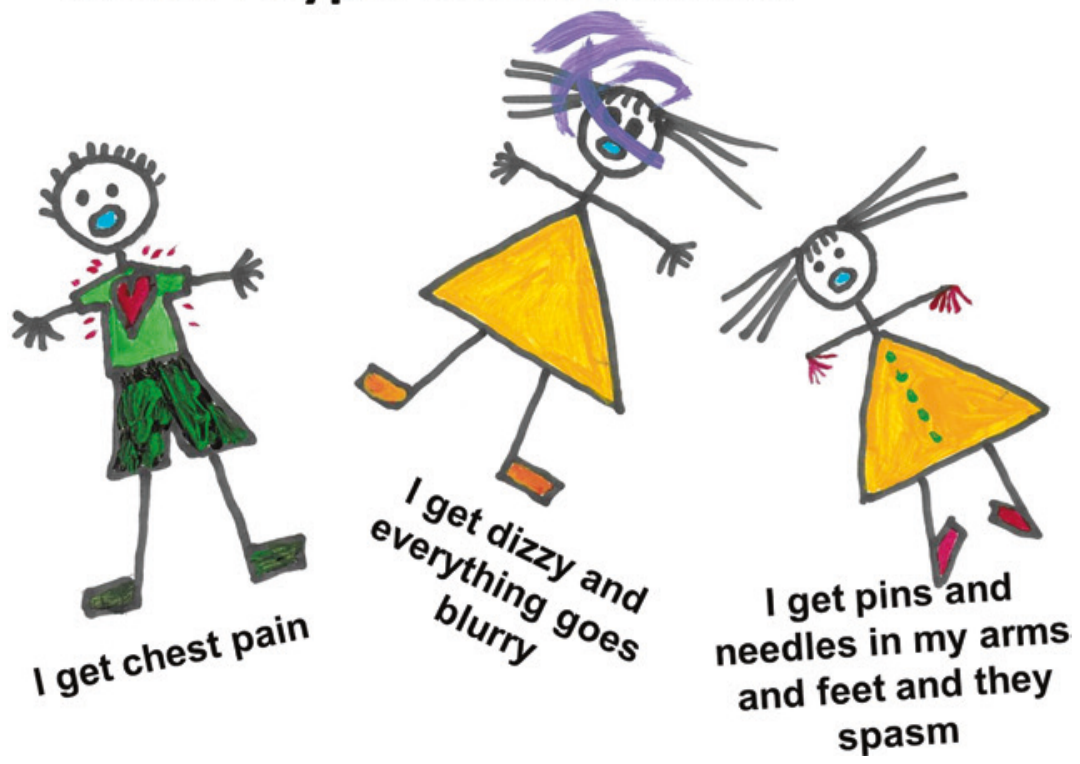

Fig. 7.3 Different body responses to hyperventilation. Individuals' physiological responses to hyperventilation vary widely, as these three examples of hyperventilation indicate. The boy on the left experiences chest pain due to constriction of cardiac arteries. The girl in the middle experiences dizziness and blurry vision due to constriction of cerebral arteries. The girl on the right experiences pins and needles (paraesthesias) and painful cramps of muscles in hands and feet (carpopaedal spasms) due to increased excitability of sensory and motor axons in the peripheral nervous system (๑ Kasia Kozlowska 2017)

(carpopedal spasms). Hyperventilation also causes vasoconstriction of arteries supplying blood and oxygen to the heart. Excessive vasoconstriction decreases blood flow and delivery of oxygen, and may cause hyperventilation-induced chest pain. Sustained increases in respiratory rate over time-which increase the body's demand for energy-will contribute to symptoms of fatigue. When talking with the family, the clinician can point out that the child's respiratory motor system is working very hard and that it is not surprising that the child feels fatigued and exhausted. 


\section{In the Brain}

Hyperventilation initially causes cortical excitation; that is, it increases brain arousal and neuron excitability. High levels of cortical arousal can impair prefrontal cortex function, shift the brain to a defensive mode of organization, and increase the probability that evolutionarily more primitive modes of neurological function will emerge (Arnsten 2015).

If hyperventilation continues beyond the short term, it causes (to an extent that varies from individual to individual) vasoconstriction of arteries supplying blood and oxygen to the brain, resulting in decreased blood flow and delivery of oxygen, and in some degree of cerebral hypoxia. Symptoms of cerebral hypoxia include dizziness, changes in vision (ranging from changes in colour to complete blackout), decreasing levels of consciousness (to loss of consciousness), loss of muscle tone (potentially culminating in falling), and so on. The prefrontal cortex is particularly sensitive both to cortical arousal and to hypoxia.

What this means clinically is that some children will have changes in consciousness with hyperventilation. In some cases, their heads will drop, and they will be 'out of it' for a few seconds; other children may faint; others may have episodes of crying and distress (loss of top-down inhibition); and still others will go into an altered, dissociated state. In extreme cases, the child may even experience an NES, with spasmodic movements (Kozlowska et al. 2017). The stressed brain undergoes a cascade of neurobiological changes that we are only just beginning to understand. Hyperventilation contributes to these changes in a major way. Importantly, interventions that target breathing and that improve regulation of the respiratory motor system provide powerful tools to help the child regulate her brain and body (see vignette of Bella, below).

Twelve-year-old Bella, whom we meet earlier in the chapter, learnt to identify body symptoms that signalled that she was beginning to hyperventilate, including not feeling real and a sense of 'spacing out', a change in the quality of auditory and visual stimuli, a cold feeling in the chest and stomach, and feeling dizzy. Bella learnt to implement a slow-breathing strategy—at the point that she first noticed the warning signs - to avert the pending NES. 


\section{Symptoms Associated with Increased Tone in Laryngeal Muscles and the Vocal Folds}

The intrinsic laryngeal muscles that are involved in voice production are striated muscles attached to cartilage (for details about striated muscles, see Online Supplement 7.1). The vocal folds-also popularly known as vocal cords - are made up of mucous membrane and intrinsic laryngeal muscle. Sound is produced when the vocal folds vibrate. In states of high arousal, sympathetic activation is coupled with increased tone in the laryngeal muscles, together with increased tone in the postural muscles of the neck, back, and legs (Helou et al. 2013, 2018; Kozlowska et al. 2015) (see also Online Supplement 7.1). This increase in laryngeal muscle tone causes an increase in the pitch of the voice, with the consequence that the vocalizations of stressed children are high in pitch. The same things happen in other mammals, for example, rat pups emit high-pitched-actually, ultrasonic_vocalizations on separation from their mothers, which the rat pups find to be very stressful (for references see Online Supplement 1.3).

Functional presentations involving the laryngeal muscles/vocal folds include functional aphonia (total loss of voice), functional dysphonia (high-pitched baby voice, whispered phonation, falsetto voice, vocal fatigue, voice instability [including a breathy, hoarse, or rough voice]), diplophonia (with two tones concurrently), and a voice that breaks uncontrollably or has a tight, strained, or strangled quality (Baker 2017).

Akin to other functional neurological symptoms, symptoms involving the laryngeal muscles/vocal folds are associated with aberrant patterns of motor activation in the brain and with aberrant activation of the brain stress systems (Dietrich et al. 2012; Roy et al. 2019) (see Chapter 10). Likewise, patients with functional voice disorders, like others with functional somatic symptoms, typically present to the health care system with a single set of functional symptoms, but, if asked, they also report other symptoms pertaining to activation of the stress system more generally (Demmink-Geertman and Dejonckere 2002; Helou et al. 2018) (see Online Supplement 7.1). 
The case of Brian is an amalgam kindly put together by our colleague Jan Baker, a speech pathologist who has particular expertise with functional voice disorders (Baker 2017).

Brian, a 15-year-old boy, presented for speech therapy assessment with a functional dysphonia of four years' duration. Even though Brian was post-pubertal-puberty had started at age 11 years-his voice pitch remained abnormally high, with marked vocal instability in the form of uncontrollable pitch breaks from falsetto phonation to a rough, diplophonic voice. Other names for this type of functional dysphonia are puberphonia and mutational falsetto. Brian had seen many speech therapists and ear, nose, and throat specialists. Repeated laryngoscopic assessments had confirmed the following: normal laryngeal and vocal-fold structures; extreme tension in the extrinsic and intrinsic laryngeal muscles; and tight sphincteric constriction of the false vocal folds, often obliterating the view and interfering with the function of his true vocal folds. A trial of botulinum toxin injection into Brian's false vocal folds had been administered on one occasion in order to elicit relaxation of this extreme pattern of supraglottic constriction, but the intervention was not effective.

On presentation, Brian appeared to be physically tense, with raised shoulders, high clavicular breathing, and a tight jaw. He appeared anxious and shy, found it difficult to make eye contact, and noted that he found his high-pitched voice both embarrassing and debilitating. He acknowledged that his parents and school teachers were concerned about the effect that his voice was having on his school attendance and also about his reticence to join in activities with other young people. His developmental history, carefully put together by the therapist, disclosed no severely stressful life events or difficulties either at the onset of puberty or over the four-year period of his dysphonia.

In the ordinary course of events, a brief, one- or two-session intervention by a speech pathologist is effective in restoring normal phonation in adolescents such as Brian. For Brian, however-because he had not disclosed the psychological stress that was driving the functional dysphonia (see below) — the intervention took months of coordinated effort involving Brian, his family, his school teachers, and two close friends. Standard behavioural techniques to facilitate normal phonation proved ineffective. The 
breakthrough came when Brian was able to experience his normal voice in association with a strongly felt emotion-in Brian's case, his laughter while watching television in the privacy of his bedroom. A strategy was devised to record Brian's voice during a particular program that he enjoyed. In the recording of Brian's free, uninhibited emotional laughter, Brian heard and recognized his normal voice at a pitch and quality appropriate to his age and maturity. The essence of this intervention was to build upon his spontaneous motor functioning (in the form of laughter), thereby changing the focus of attention and releasing his voice from cortical, or 'top-down', control and interference. It was only after many months of therapy-with Brian being held in both a trusting therapeutic relationship and trusting relational network (family, teachers, and friends) while searching for his true voice - that he was able to raise and address issues that had been troubling him since the age of 12. Ever since his voice started to break (at puberty), Brian had held fears that he might be gay — which he now felt able to discuss.

In rare cases, an overly robust motor response in the larynx can cause complete adduction of the vocal folds, ventricular bands, and supraglottic structures, thereby closing the airway, cutting off or reducing oxygen supply, and causing hypoxia. Potential sequelae include a change in consciousness, fainting, or fainting followed by a hypoxia-related NES. In some cases, this sphincteric vocal fold adduction is misdiagnosed as asthma. For clinical vignettes detailing atypical, stress-related motor responses in the larynx, see Kozlowska and colleagues (2018a, b).

Mika was a 9-year-old boy with a history of chronic, treatment-resistant asthma and weekly presentations to hospital. He was very anxious about his asthma attacks. Sometimes when Mika thought that he was having an asthma attack, he would begin to panic, his heart would race, and he would begin to breath quickly, coughing intermittently and taking in huge, noisy gulps of air. In this state of high arousal, his vocal folds would adduct, thereby closing his air passage. During one such event, the respiratory specialist visualized the adduction of the vocal folds using a laryngoscope. Mika’s noisy breathing would suddenly stop, his eyes would roll back — a blue tinge around his lips signalling hypoxia — and he would slump to the side. Sometimes Mika would be incontinent of urine, and sometimes his body would jerk and shake (a hypoxia-related NES). (This vignette was first presented in Kozlowska et al. 2018a.) 


\section{Symptoms Associated with Muscles of the Inner Ear}

The muscles of the inner ear-the tensor tympani and stapedius-are the smallest striated muscles in the body. Contracting in response to noise, they inhibit, through what is known as the acoustic reflex, the vibrations of the small bones in the middle ear and thereby inhibit, in turn, the transmission of sound. In children, persisting tinnitus or sound sensitivity is often triggered by physical stress (e.g., attendance at a loud concert) or infection (e.g., a viral infection). Akin to other functional somatic symptoms, aberrant motor patterns that maintain tinnitus appear to be maintained by ongoing activation of the brain stress systems (Chen et al. 2017; Leaver et al. 2011) (see Chapter 11). For vignettes see Louisa in Chapter 2 and Bellynda in Chapter 8.

\section{Symptoms Associated with Muscles Involved in Coughing}

Coughing is a motor pattern that involves muscles of the larynx, pharynx, chest, and diaphragm. Cough is a defensive reflex that 'preserves the gas-exchanging functions of the lung by facilitating clearance of aspirate, inhaled particulate matter, accumulated secretions, and irritants that are either inhaled or formed at sites of mucosal inflammation' (Canning et al. 2014, p. 1633). The cough reflex can be triggered by activation of parasympathetic (vagal) afferents from the larynx, trachea, and bronchi, as well as by irritation of the lower oesophagus (in a subgroup of individuals) or by mechanical stimulation of the ear (mechanical stimulation of afferent vagal fibres in the external auditory canal can activate the cough reflex in another subgroup of individuals). Functional cough (also known as habit or psychogenic cough) or throat clearing usually develops in the wake of an infection involving the pharynx or the bronchi. Like other functional disorders, functional cough is an aberrant motor pattern that is presumably maintained by ongoing activation of the brain stress systems (Canning et al. 2014). The symptoms of functional cough may range 
from mild coughing to intense, almost constant coughing that resembles a goose honking or a dog or seal barking. The third author $(\mathrm{HH})$ had a patient who had been 'barking' almost continuously for more than a year.

\section{Symptoms Associated with Muscles Involved in Swallowing, Belching, and Rumination}

Functional somatic symptoms can also occur in connection with motor patterns that involve the striated (skeletal) muscles in the throat, neck, and chest (innervated by somatomotor nerves) and smooth muscle in the upper gastrointestinal tract (innervated by the autonomic nervous system). These muscles work together to enable swallowing, belching, and rumination. Gastroenterologists commonly refer to the functional somatic symptoms associated with these processes as (aberrant) behaviours, habits, or reflex responses that develop as reactions to stimuli such as food, infection, strong emotions, or even acute work pressure. Functional disorders associated with swallowing and belching (and unrelated to any organic pathology) include globus sensation (the feeling of having a lump in the throat), excessive belching (bringing up air), rumination/regurgitation (bringing up food, in a process similar to belching), and other unwanted action patterns mediated by this group of muscles. Akin to other functional somatic symptoms, these motor patterns appear to be maintained by ongoing activation of the brain stress systems (Tornblom and Drossman 2018) (see Chapter 11).

For more information-and references-about the mechanics (muscle patterns) in belching, rumination/regurgitation, and globus sensation, see Online Supplement 7.1.

Functional symptoms involving muscles of breathing and swallowing can present as lone symptoms (see case of Fiona in Kozlowska [2013]), as a cluster of symptoms involving breathing or swallowing functions, or, as in the following case of Maddie, alongside other functional neurological symptoms.

Maddie, a 15-year-old girl—the youngest of six children—attended a special school for the intellectually impaired. Maddie's family split their 
time between the farm (run by the family, and now her father, for generations) and the city (where Maddie lived with her mother, whose chronic medical condition required her close proximity to the hospital). Despite her intellectual disability, Maddie had done well because she had always been supported by her siblings. But when Maddie turned 15, her sisterthe last sibling living in her mother's house-left home to get married, leaving Maddie responsible, all on her own, for managing the house, the physical and emotional needs of her ill mother, and the continuing demands of her own schooling. Three months into this new arrangement, Maddie experienced a fall, knocking her head and hurting her right knee. In the very short term, she suffered from a loss of memoryshe no longer remembered that her sister had left home-and also from pain in her right knee. Over the next month, the memory loss did not resolve, and the pain spread to involve Maddie's whole leg. After seeing many doctors, who could find no medical explanation for her problems, Maddie was referred to the complex pain clinic.

Because the farm work was at its peak in and around this time period, the visits from those living on the farm-Maddie's father and some of her siblings - were brief, and Maddie became more and more distressed. Three months after her fall, she began to complain of a lump in the throat that fluctuated in intensity, that came and went, and that, when present, made it difficult to swallow food. Maddie also began to experience episodes of panic: breathlessness, dizziness, blurred vision, sweatiness, a pounding heart, and tingling in the fingers, feet, neck, back, shoulders, and left side of the face. During these episodes, Maddie's respiratory rate would reach 40 breaths per minute, which she was unable to lower. The panic attacks soon morphed into NES, which sometimes lasted over an hour (Kozlowska et al. 2018a). During the NES, Maddie was unresponsive, and her body shook and jerked. Sometimes she was aware of what people said around her, and sometimes not. Following one prolonged NES, Maddie experienced a range of other functional neurological symptoms that took weeks to resolve: she lost power in her legs and could no longer feel them, was unable to void her bladder, lost her capacity to talk, and had difficulty swallowing, with the consequence that saliva dribbled from the corner of her mouth. After substantial work regarding regulation strategies and some changes in the family situation, Maddie regained use of her legs, bladder, voice, and swallowing, and joined her father and siblings on the farm. Because her NES had not yet 
resolved, she initially wore a bike helmet when mobilizing around the property. Twelve months later, all her symptoms had fully resolved, and Maddie was happily contributing to the running of the family farm, with intermittent visits to her mother in the city.

\section{Symptoms Associated with Muscles in the Bladder Sphincter}

The skeletal muscle in the bladder sphincter is innervated by somatomotor nerves. The smooth muscle in the bladder sphincter is innervated by sympathetic (visceromotor) nerves, and the smooth muscle in the bladder wall is innervated by parasympathetic (visceromotor) nerves. Urination is a coordinated response involving (1) inhibition of sympathetic efferents and relaxation of smooth muscle fibres in the urethral sphincter, (2) inhibition of somatic efferents and relaxation of striated muscle fibres in the urethral sphincter, and (3) activation of efferent parasympathetic fibres and concomitant contraction of the bladder wall. Because the skeletomotor and autonomic (sympathetic) systems are coupled, stress-related activation of the sympathetic efferents coupled with activation of somatomotor efferents to the sphincter may be responsible for stress-related (functional) bladder retention. For brain regions involved in urination, see references in Online Supplement 1.3.

\section{Symptoms That Mimic Neurological Diseases}

For completeness, we mention symptoms that mimic neurological conditions such as stroke, epilepsy, or infections of the brain. Common symptoms include weakness or paralysis of the legs or arms, loss of coordination of the legs, and contraction of muscles, causing abnormal posture of the limbs (dystonia). We discuss these presentations in more detail in Chapter 11. Neurologists consider these symptoms under the umbrella of FND. Some presentations associated with increased tone in laryngeal muscles and the vocal folds are also included under FND. 
Likewise, bladder retention sometimes occurs alongside other FND symptoms and may be included under FND because it mimics neurological conditions involving the spinal cord.

\section{The Fascia}

Fascia-connective tissue-is present throughout the body (Stecco 2015). Superficial fascia refers to a fibrous layer that lies within the skin and that covers the body. This fascia protects the body as a whole. Deep (muscular) fascia 'refers to all of the well-organized, dense, fibrous layers that interpenetrate and surround muscles, bones, nerves and blood vessels, binding all of these structures together into a firm, compact, continuous mass' (Stecco et al. 2016, p. 162). This fascia helps maintain posture and supports body movements. Visceral fascia refers to the layers of connective tissue that surround, wrap, and help suspend the organs and tissues. This fascia plays a role in maintaining homeostasis- the internal environment of the body.

The fluidity or rigidity of the fascia system is linked to the child's sense of health and well-being. Healthy interactions between the child and the attachment figure, including physical contact that modifies the fascia system-for example, soothing touch such as holding the child tight or stroking the child's body-will simultaneously settle the autonomic system, deactivate the skeletomotor system, and modify both the pliability and texture of the fascia, contributing to the child's sense of physical and emotional well-being (Schleip 2003; Schleip and Klingler 2019). Likewise, many of us find that therapeutic touch or physical palpation techniques help release tension patterns in our own bodies.

By contrast, activation of the autonomic system, with an increase in sympathetic arousal, is typically accompanied by increased activity of skeletal muscle and increased tension within the fascia system. Presumably, these increases, if chronic, will contribute both to aberrant motor patterns that present as functional somatic symptoms and to symptoms of pain that accompany some of these patterns. For example, we can hypothesize that the recurrent abdominal and mediastinal pain 
(precordial catch syndrome) experienced by 15-year-old Evie (whom the reader met in Chapter 2) involved fascial tension patterns, much like the mechanisms that are hypothesized for chronic urogenital pain in women (Jantos, forthcoming 2020; Jantos and Stecco, forthcoming 2021).

In addition, because immune-inflammatory cells also reside within the fascia and because the fascia is rich is nerve receptors that carry interoceptive information (e.g., pain), activation of immune-inflammatory cells by stress (physical or psychological) is likely to contribute to chronic pain by secreting pro-inflammatory molecules that activate pain neurons from within the fascia (Jantos, forthcoming 2020) (see also Chapter 9).

For a simple-to-read article about fascia and references to science articles, see Online Supplement 7.1.

In this chapter we have examined the many different functional somatic symptoms that involve aberrant activation of the skeletomotor system. We have seen that the skeletomotor system works in close collaboration with the autonomic nervous system-increases in arousal are accompanied by activation of skeletal muscles-and that aberrant activation of the skeletomotor system/motor systems in the brain is maintained by activation of the brain stress systems. In the face of stress, whether physical or psychological, both systems activate together to manage threat. When these processes go awry-when the interplay between these systems becomes maladaptive-aberrant motor patterns that present as functional somatic symptoms may arise. In Chapter 8 we look at the role of the hypothalamic-pituitary-adrenal (HPA) axis in functional somatic symptoms.

\section{References}

Arnsten, A. F. (2015). Stress Weakens Prefrontal Networks: Molecular Insults to Higher Cognition. Nature Neuroscience, 18, 1376-1385.

Baker, J. (2017). Psychosocial Perspectives on the Management of Voice Disorders. Oxford: Compton. 
Canning, B. J., Chang, A. B., Bolser, D. C., Smith, J. A., Mazzone, S. B., Mcgarvey, L., et al. (2014). Anatomy and Neurophysiology of Cough: Chest Guideline and Expert Panel Report. Chest, 146, 1633-1648.

Chen, Y. C., Xia, W., Chen, H., Feng, Y., Xu, J. J., Gu, J. P., et al. (2017). Tinnitus Distress Is Linked to Enhanced Resting-State Functional Connectivity from the Limbic System to the Auditory Cortex. Human Brain Mapping, 38, 2384-2397.

Chudleigh, C., Savage, B., Cruz, C., Lim, M., McClure, G., Palmer, D. M., et al. (2019). Use of Respiratory Rates and Heart Rate Variability in the Assessment and Treatment of Children and Adolescents with Functional Somatic Symptoms. Clinical Child Psychology and Psychiatry, 24, 29-39.

Demmink-Geertman, L., \& Dejonckere, P. H. (2002). Nonorganic Habitual Dysphonia and Autonomic Dysfunction. Journal of Voice, 16, 549-559.

Dietrich, M., Andreatta, R. D., Jiang, Y., Joshi, A., \& Stemple, J. C. (2012). Preliminary Findings on the Relation Between the Personality Trait of Stress Reaction and the Central Neural Control of Human Vocalization. International Journal of Speech-Language Pathology, 14, 377-389.

Dum, R. P., Levinthal, D. J., \& Strick, P. L. (2016). Motor, Cognitive, and Affective Areas of the Cerebral Cortex Influence the Adrenal Medulla. Proceedings of the National Academy of Sciences of the United States of America, 113, 9922-9927.

Fleming, S., Thompson, M., Stevens, R., Heneghan, C., Plüddemann, A., Maconochie, I., et al. (2011). Normal Ranges of Heart Rate and Respiratory Rate in Children from Birth to 18 Years of Age: A Systematic Review of Observational Studies. Lancet, 19, 1011-1018.

Helou, L. B., Rosen, C. A., Wang, W., \& Verdolini Abbott, K. (2018). Intrinsic Laryngeal Muscle Response to a Public Speech Preparation Stressor. Journal of Speech, Language, and Hearing Research, 61, 1525-1543. Helou, L. B., Wang, W., Ashmore, R. C., Rosen, C. A., \& Abbott, K. V. (2013). Intrinsic Laryngeal Muscle Activity in Response to Autonomic Nervous System Activation. Laryngoscope, 123, 2756-2765.

Jafari, H., Courtois, I., Van Den Bergh, O., Vlaeyen, J. W. S., \& Van Diest, I. (2017). Pain and Respiration: A Systematic Review. Pain, 158, 995-1006. Jantos, M. (forthcoming 2020). A Myofascial Perspective on Chronic Urogenital Pain in Women. In G. A. Santoro, A. P. Wieczorek, \& A. H. Sultan (Eds.). Pelvic Floor Disorders: A Multidisciplinary Textbook (2nd ed.). Springer. 
Jantos, M. \& Stecco, C. (forthcoming 2021). Fascia of the Pelvic Floor. In R. Schleip, M. Driscoll, C. Stecco, \& P. Huijing (Eds.), Fascia: The Tensional Network of the Human Body (2nd ed.). London: Elsevier.

Kain, K. L., \& Terrell, S. J. (2018). Nurturing Resilience: Helping Clients Move Forward from Developmental Trauma: An Integrative Somatic Approach. Berkeley, CA: North Atlantic Books.

Koizumi, K., \& Brooks, C. M. (1972). The Integration of Autonomic System Reactions: A Discussion of Autonomic Reflexes, Their Control and Their Association with Somatic Reactions. Ergebnisse der Physiologie (Reviews of Physiology), 67, 1-68.

Kozlowska, K. (2013). Stress, Distress, and Bodytalk: Co-constructing Formulations with Patients Who Present with Somatic Symptoms. Harvard Review of Psychiatry, 21, 314-333.

Kozlowska, K., Chudleigh, C., Cruz, C., Lim, M., McClure, G., Savage, B., et al. (2018a). Psychogenic Non-epileptic Seizures in Children and Adolescents: Part I-Diagnostic Formulations. Clinical Child Psychology and Psychiatry, 23, 140-159.

Kozlowska, K., Chudleigh, C., Cruz, C., Lim, M., McClure, G., Savage, B., et al. (2018b). Psychogenic Non-epileptic Seizures in Children and Adolescents: Part II-Explanations to Families, Treatment, and Group Outcomes. Clinical Child Psychology and Psychiatry, 23, 160-176.

Kozlowska, K., Rampersad, R., Cruz, C., Shah, U., Chudleigh, C., Soe, S., et al. (2017). The Respiratory Control of Carbon Dioxide in Children and Adolescents Referred for Treatment of Psychogenic Non-epileptic Seizures. European Child and Adolescent Psychiatry, 26, 1207-1217.

Kozlowska, K., Walker, P., McLean, L., \& Carrive, P. (2015). Fear and the Defense Cascade: Clinical Implications and Management. Harvard Review of Psychiatry, 23, 263-287. https://www.ncbi.nlm.nih.gov/pmc/articles/ PMC4495877/.

Leaver, A. M., Renier, L., Chevillet, M. A., Morgan, S., Kim, H. J., \& Rauschecker, J. P. (2011). Dysregulation of Limbic and Auditory Networks in Tinnitus. Neuron, 69, 33-43.

Levine, P. (1997). Waking the Tiger: Healing Trauma. Berkeley, CA: North Atlantic Books.

Ogden, P., \& Fisher, J. (2015). Sensorimotor Psychotherapy: Interventions for Trauma and Attachment. New York: Norton.

Payne, P., Levine, P. A., \& Crane-Godreau, M. A. (2015). Somatic Experiencing: Using Interoception and Proprioception as Core Elements of Trauma Therapy. Frontiers in Psychology, 6, 93. 
Roy, N., Dietrich, M., Blomgren, M., Heller, A., Houtz, D. R., \& Lee, J. (2019). Exploring the Neural Bases of Primary Muscle Tension Dysphonia: A Case Study Using Functional Magnetic Resonance Imaging. Journal of Voice, 33, 183-194.

Schleip, R. (2003). Fascial Plasticity-A New Neurobiological Explanation: Part 1. Journal of Bodywork and Movement Therapies, 7, 11-19.

Schleip, R., \& Klingler, W. (2019). Active Contractile Properties of Fascia. Clinical Anatomy, 32, 891-895.

Simons, D. G., Travell, J., \& Simons, L. S. (1999). Myofascial Pain and Dysfunction: The Trigger Point Manual. Baltimore, MD: Williams \& Wilkins.

Stecco, A., Stern, R., Fantoni, I., De Caro, R., \& Stecco, C. (2016). Fascial Disorders: Implications for Treatment. Physical Medicine and Rehabilitation, 8, 161-168.

Stecco, C. (2015). Functional Atlas of the Human Fascial System. Edinburgh: Elsevier.

Tornblom, H., \& Drossman, D. A. (2018). Psychotropics, Antidepressants, and Visceral Analgesics in Functional Gastrointestinal Disorders. Current Gastroenterology Reports, 20, 58.

Westgaard, R. H., Mork, P. J., Loras, H. W., Riva, R., \& Lundberg, U. (2013). Trapezius Activity of Fibromyalgia Patients Is Enhanced in Stressful Situations, but Is Similar to Healthy Controls in a Quiet Naturalistic Setting: A Case-Control Study. BMC Musculoskeletal Disorders, 14, 97. 
Open Access This chapter is licensed under the terms of the Creative Commons Attribution-NonCommercial-NoDerivatives 4.0 International License (http://creativecommons.org/licenses/by-nc-nd/4.0/), which permits any noncommercial use, sharing, distribution and reproduction in any medium or format, as long as you give appropriate credit to the original author(s) and the source, provide a link to the Creative Commons license and indicate if you modified the licensed material. You do not have permission under this license to share adapted material derived from this chapter or parts of it.

The images or other third party material in this chapter are included in the chapter's Creative Commons license, unless indicated otherwise in a credit line to the material. If material is not included in the chapter's Creative Commons license and your intended use is not permitted by statutory regulation or exceeds the permitted use, you will need to obtain permission directly from the copyright holder.

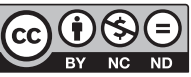

